\title{
Genetics In Pediatric Dentistry -A Review
}

\author{
Drifzah $^{1}$, Dr.DivyaPrahlad ${ }^{2}$, Dr.Priya Subramaniam ${ }^{3}$, Dr.Kinjal Shah ${ }^{4}$ \\ ${ }^{1}$ Consulting Pedodontist, Maya Cleft Center, Srinagar. \\ ${ }^{2}$ Pedodontist, Ministry of Interior Dental Department, State of Oman. \\ ${ }^{3}$ Head, Professor and Principal, The Oxford Dental College and Hospital, Bangalore. \\ ${ }^{4}$ Senior Lecturer, Manubhai Patel Dental College, Vadodara.
}

\begin{abstract}
Genetics is the science of heredity and variation. It plays an important role in determining our individuality. The term "genetics" conveys two different concepts: genetics as the study of inherited characteristics, and genetics as the study of cellular processes controlled by DNA.Developmental defects of teeth can occur as isolated genetic traits, be associated with a chromosomal abnormality or syndrome, or be inherited as a complex trait with genetic and environmental interactions. Numerous hereditary syndromes are associated with congenitally missing teeth. Often pediatric dentists are the first health care practitioners to document dysmorphic features in a child. It is important for them to have an understanding of molecular genetics because the sensitivity and specificity of molecular-based diagnostics have revolutionized how diseases and disorders are defined.
\end{abstract}

Keywords: Genetics, Caries, Genes, Mendel.

\section{INTRODUCTION}

Genetics is a science of potentials. It deals with the transfer of biological information from cell to cell, from parents to offspring, and thus from generation to generation. Genetics has revealed that any two individuals share $99.9 \%$ of their DNA sequences. Thus, the remarkable diversity of humans is encoded in about $0.1 \%$ of our DNA. Genetics has revealed that any two individuals share $99.9 \%$ of their DNA sequences.

\section{Historical Perspective}

According to Stent (1971) the first evidence of inheritance was taught and developed by Hippocrates in fifth century BC in Greece. Hippocrates ideas can be termed as 'bricks and mortar theory' which states that hereditary material consists of physical matter. He postulated that elements from all part of the bodybecame concentrated in male semen and then formed into a human in the womb. He also believed in the inheritance of acquired characteristics. A century later Aristotle criticized Hippocrates theory and instead proposed that heredity involved the transmission of information-'a blueprint model'. Aristotle discarded Hippocrates theory for several reasons. He pointed out that individuals sometimes resemble remote ancestors rather than their immediate parents.

Genetics did not develop suddenly. Instead it evolved from intellectual background of Darwin's time that dates back to 1859. Darwin proposed that a species changes as a result of generations of competition among individuals. Within a species individuals vary with respect to heritable characteristics that influence the ability to survive and reproduce.

Gregor Mendel (1822-1884) is appropriately called as the "Father of genetics". His precedent- setting experiments with garden peas were published in 1866. Although Mendel devised a precise mathematical pattern for the transmission of hereditary units, he had no concept of biological mechanisms involved. August Weismann (1834-1914) gave the germ-plasm theory which stated that the germ line is the continuous element, and the successive bodies of higher animals and plants are side branches budded off from it, generation after generation.

Galton showed that, on the average, an individual inherits $1 / 4$ of his characteristics from each parent, 1/16 from each grandparent, 1/64 from each great-grandparent, and so on. In 1930, G.W. Beadle, B. Ephrussi, E. L. Tatum, J. B. S. Haldane hypothesized that: The gene was at first characterized as an indivisible unit of structure, unit of mutation and unit of function with all three of these attributes considered equivalent. The concept given by them is called one gene-one enzyme concept. ${ }^{1}$

Concept of the gene has evolved from Mendel's "unit factor" controlling on a phenotypictrait to the unit of genetic material specifying one polypeptide and operationally defined by the complementation test. There has been no change in the concept of the gene as the basic unit of function since its discovery by Mendel in $1866 .{ }^{2}$ The discoveries of the mid to late 20th century defined processes that would provide the tools for molecular biology, recombinant DNA technology, and finally the biotechnology industry. Restriction enzymes were discovered and used to construct recombinant DNA molecules. The advent of protein and DNA 
sequencing launched a new era of phylogenetics. Species could now be compared at the molecular level. The information age is essential to genomics. The electronic analysis, distribution and storage of genomic data are a hallmark of the science. The internet spawned the distribution of information from central databases. E-mail connected scientists and fostered the rapid exchange of ideas. The advent of the WWW provided a new medium for the presentation of information. ${ }^{3}$

\section{Fundamentals of Genetics}

The genome contains the entire genetic content of a set of chromosomes present within a cell or an organism. Within genome are genes that represent the smallest physical and functional units of inheritance that resides in specific sites called "loci" or "locus" for a single location. The term gene was coined by Wilhelm Johannsen in 1909. The gene was first defined as the unit of genetic information that controls a specific aspect of the phenotype. At a more fundamental level the gene has been defined as the unit of genetic information that specifies the synthesis of one polypeptide. ${ }^{3}$

A gene can be defined as the entire DNA sequence necessary for the synthesis of a functional polypeptide molecule (production of a protein via a messenger RNA intermediate) or RNA molecule (transfer RNA and ribosomal RNA)Operationally, the gene includes the $5^{\prime}$ and $3^{\prime}$ non coding regions that are involved in regulating the transcription and translation of the gene and all introns within the gene. The structural gene refers to the portion that is transcribed to produce the RNA product. ${ }^{4}$

\section{Functions of Genes}

Genes accomplish their function:

1. Through replication that result in more units like themselves

2. Through transcription and translation, whereby proteins that function as determiners in metabolism of cell are synthesize

3. Genes act by determining the structure of proteins, which are responsible for directing cell metabolism through their activity as enzymes. ${ }^{5}$

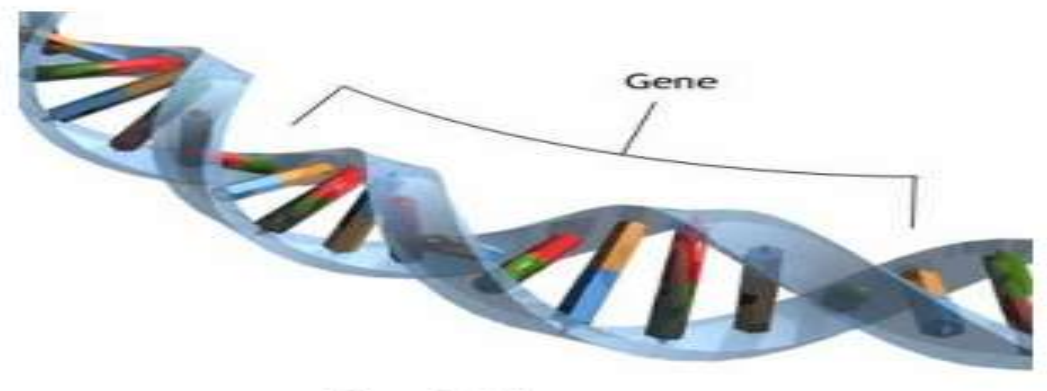

\section{GENETICS AND DENTAL CARIES}

Dental caries is a disease of the dental hard tissues, characterized initially by the decalcification of the inorganic portions of the tooth. Loss of the mineral content is then followed by the breakdown of the organic matrix.

\section{Genetics In Dental Caries Through Twin Studies}

Studies of the etiology of dental caries suggest that both genetic and environmental factors may be concerned with the development of dental caries. Several major genes have been identified which condition defective formation of enamel or dentine leading to development of rampant caries in such teeth. ${ }^{6}$ Twin studies have provided strong evidence for the role of inheritance.Goldberg found that identical twins showed decay in corresponding teeth and heredity affects dental decay only in as much as it controls the shape of a tooth and its pits and fissures and its position in the dental arch. ${ }^{7}$

Bordoni concluded that there is a "strong genetic component in primary teeth which affects the incidence of caries and has shown the association of TAS2R gene with caries in primary dentition. ${ }^{8}$

Mansbridge concluded from his study that "Environmental factors clearly have greater influence but the genetic factors also contribute to the causation of dental caries". ${ }^{9}$ This led to the conclusion of Finn and Caldwell that, "Equal genetic weight should not be ascribed to both types of lesions that are smooth surface lesions and pit and fissure lesions". 10

Boraas from his study in which twins were reared apart speculated on the particular inherited traits that could contribute to the results by stating "Several genetically variable factors which may be involved in the 
development of dental caries and contribute to the greater monozygotic similarity in dental caries experience are, 1) Salivary factors and oral flora, 2) tooth eruption time and sequence, 3) tooth morphology, 4) arch shape, 5) dental spacing, 6) propensity for diet." ${ }^{11}$ Klein and Palmer" ${ }^{12}$ and Klein's ${ }^{13}$ findings indicated that children have a caries experience remarkably similar to that of their parents when the susceptibility of both parents is the same (either high or low). When caries susceptibility of the two parents is dissimilar, however, the children's susceptibility tends to be more like that of the mother than that of the father. This finding was particularly evident in daughters.Li and Caulfield found that mothers are the principle source of streptococci mutans to their infants, with a greater rate of transmission to female than male infants. The high degree of fidelity between strains of MS in mothers and their female infants in contrast to those isolated from male infants, indicates that the conservation of MS within mother-infant pairs is gender specific. It has been observed that infants whose teeth emerged early were significantly more likely to acquire MS from their mothers than infants whose teeth emerged later. That tooth emergence in females occurs sooner than in males is commonly accepted. Children whose teetherupt earlier than the expected time are more advanced in other ways that might influence fidelity of acquisition, such as a more developed immune system. The obvious explanation is that mothers enjoy the most contact with their infants and, therefore, constitute the major source of MS. The mechanism may be that intimacy between a mother and her spouse results in the appearance of an immunological recognition of the father's indigenous bacteria as non-self, and this immunological awareness is transferred to the infant through the placenta or breast milk. Mothers transfer to their infants not only maternal immunoglobulins via the placenta and colostrum, but also a complementary set of indigenous bacteria capable of co-existing with these maternally-derived or directed immunity factors. ${ }^{14}$

\section{Genes And Saliva}

Human saliva is a complex mixture of proteins. Kauffman and Keller estimated that as much as twothirds of parotid salivary proteins belong to a class designated as proline-rich proteins (PRP) due to high content of the amino acid proline. The human salivary PRPs are determined by six closely linked genes on chromosome $12 \mathrm{pl} 3$ that can be divided into two major families based on the sensitivities of the DNA sequence to the restriction enzymes HaeIII and BstNI. ${ }^{15}$

\section{Genetic Modification Of Dental Enamel Altering Susceptibility To Dental Caries}

Tooth enamel is a composite bioceramic composed largely of carbonated hydroxyapatite, Hap, and very small amounts of protein remnants. Tooth enamel is the most durable of all tissues surviving for millennia as long as it is not exposed to carious acid attack.

Amelogenin, the protein product of the AMELX Xp22.3- p22.1 and AMELY Yp11 genes, is considered to be critical for normal enamel thickness and structure. Amelogenin is a likely candidate gene for caries susceptibility in humans and has differential expression in males versus females. Mutations and deletions in amelogenin cause one of the forms of X-linked amelogenesisimperfecta. Ameloblastin is expressed during the differentiation of inner enamel epithelium into ameloblasts, with intense localization in the Tomes processes of secretory ameloblasts. Genetic variation in these genes contributes to structural alterations of the enamel that may cause higher levels of mineral losses under acidic conditions and/or facilitate bacterial attachment and biofilm deposition. A locus on chromosome Xq27.1 was suggested to be harboring a protective caries gene.

A dentin gene, dentin sialophosphphoprotein (Dspp), is transiently expressed in early-stage,secretory ameloblasts at the time of DEJ formation. This is consistent with Dspp having a role in producing specialized first-formed harder enamel adjacent to the DEJ. The expression of "odontoblast" proteins such as DSPP and DMP1, at the time the DEJ is formed, appears to be products of both odontoblasts and ameloblasts. ${ }^{16}$

\section{Taste Genes Associated With Dental Caries}

Phenotypic inversions and other age-related biological responses have been characterized for TAS2R38 haplotypes or chemically determined bitter sensitivity, including correlations to food preferences, liking, and use, including sweet preferences. Bitter sensitivity of the TAS2R38 supertaster haplotype declines with age, including a substantial transition during puberty. TAS2R 38 has been correlated with caries in the primary dentition. The proline, alanine, and valine combination (PAV) represented by the nucleotides (GGC) is associated with bitter sensitivity or "supertasters", while the alanine, valine, and isoleucine combination (AVI) represented by the nucleotides (CAT) is associated with bitter insensitivity or "non-tasters". A significant association of the $\mathrm{G}, \mathrm{G}$, and $\mathrm{C}$ individual alleles representing the $\mathrm{P}, \mathrm{A}$, and $\mathrm{V}$ substitutions of supertasters with caries protection in the "primary" group has been seen. ${ }^{17}$ 


\section{GENETICS AND CHROMOSOMAL DISORDERS}

When a child is developing before birth, a minute malfunction in DNA replication or chromosome formation can result in an array of genetic disorders

Genetic diseases are produced by simple or complex mechanisms. Genetic diseases can be categorized as:

1. Chromosomal disorders, caused by abnormality in chromosome number or structure.

2. Mendelian disorders caused by abnormality of a single gene.

3. Non Mendelian disorders caused by mitochondrial mutations or altered gene expression resulting from imprinting.

Chromosomal disorders are congenital or acquired. Acquired chromosomal disorders are important in the pathogenesis and evolution of hematologic and solid organ malignancies.Congenital chromosomal disorders occur in approximately 1 in 150 live births; they with the exception of $\mathrm{X}$ and $\mathrm{Y}$ chromosomal disorders occur in pediatric age group. Such chromosomal disorders are also known as constitutional chromosomal disorders as they involve every cell of the body.Some of the congenital chromosomal disorders are listed below:

\section{$>$ Trisomy 21 Syndrome}

Down's syndrome, or mongolism, is one of the most common recognizable malformation syndromes. In 1866, Langdon Down published an essay in England in which he described a set of children with common features who were distinct from other children with mental retardation that he named "Mongolian idiocy". In the 1970s, an American revision of scientific terms changed it simply to "Down syndrome". Down's syndrome is seen in 1/650 live births. The various physical features recognized are short stature, flat occiput, epicanthic folds, broad nasal bridge, folded ears, small maxilla, protruding tongue, short hands and fingers, clinodactyly, simian line, congenital heart disease, and a gap between first and second toes. These patients have hypotonia and mental retardation. ${ }^{18}$

Dental findings found in Down's syndrome patients are late eruption of deciduous teeth, early shedding of deciduous teeth, defective or absent upper lateral incisors, anomalies of tooth shape, periodontal diseases, malocclusion, and prognathism. Periodontal disease is more prevalent problem in Down's syndrome than dental caries. Severity of tooth wear (both attrition and erosion) was significantly greater in children with Down's syndrome than in children without the syndrome. Some babies are born with heart defects and others aren't. Some children have associated illnesses such as epilepsy, hypothyroidism or celiac disease, and others don't. The first possible reason is the difference in the genes that are triplicated. The second reason that might be involved is called "penetrance." If one allele causes a condition to be present in some people but not others, it is called "variable penetrance," and that appears to be what happens with trisomy 21: the alleles don't do the same thing to every person who has it. Both reasons may be why thereis such variation in children and adults with Down's syndrome. ${ }^{19}$

\section{Cytogenetics}

Genes that may have input into Down syndrome include:

- Superoxide Dismutase (SOD1) — over expression may cause premature aging and decreasedfunction of the immune system; its role in Senile Dementia of the Alzheimer's type ordecreased cognition is still speculative.

- COL6A1 - Over expression may be the cause of heart defects

- ETS2 - Over expression may be the cause of skeletal abnormalities

- CAF1A - Over expression may be detrimental to DNA synthesis

- Cystathione Beta Synthase (CBS) - Over expression may disrupt metabolism and DNA repair

- DYRK - Over expression may be the cause of mental retardation

- $\quad$ CRYA1 - Over expression may be the cause of cataracts

- $\quad$ GART - Over expression may disrupt DNA synthesis and repair

- IFNAR -- the gene for expression of Interferon, Over expression may interfere with the immune system as well as other organ systems

Other genes that are also suspects include APP, GLUR5, S100B, TAM, PFKL. ${ }^{20}$ The risk oftrisomy 21 increases with maternal age. The birth prevelance is $0.9 / 1000$ when the mother is less than 33 years of age, $2.8 / 1000$ when the mother is 35 to 38 years old, and 38/1000 when mother is 44 years old or older.

> Cherubism (Hereditary fibrous dysplasia of the jaws, Familial intraosseous swellings of the jaws, Familial multilocular cystic disease of the jaws).

Cherubism is a hereditary condition that produces firm, painless swellings that occur bilaterally in the jaws, especially over the mandibular angles. This autosomal dominant disease is usually detected between the ages of 2 and 7 years. Cherubism or multilocularcystic disease of the jaws was first recognized as a separate entity in 1933 by William A.Jones in a family with several affected members. Cherubism is defined by the 
appearance of symmetrical, multilocular, expansile radiolucent lesions of the mandible and/or the maxilla. Swelling of submandibular lymph nodes in the early stages contributes to the fullness of the face. Cherubismlesions are limited to the jaws and in most cases the dysplastic expansile masses begin to regress with the onset of puberty. Grading systems for cherubism have been suggested to describe location and severity of lesions. $^{21}$

Cherubism grading system according to Motamedi (1998) and Raposo-Amaral (2007) ${ }^{22}$

Grade I

Lesions of the mandible without signs of root resorption

Class 1 Solitary lesion of the mandibular body

Class 2 Multiple lesions of the mandibular body

Class 3 Solitary lesion of the ramus

Class 4 Multiple lesions of the rami

Class 5 Lesions involving the mandibular body and rami

Grade II

Lesions involving the mandible and maxilla without signs of root resorption

Class 1 Lesions involving the mandible and maxillary tuberosities

Class 2 Lesions Involving the mandible and anterior maxilla

Class 3 Lesions involving the mandible and entire maxilla

Grade III

Aggressive lesions of the mandible with signs of root resorption

Class 1 Solitary lesion of the mandibular body

Class 2 Multiple lesions of the mandibular body

Class 3 Solitary lesion of the ramus

Class 4 Multiple lesions of the mandibular rami

Class 5 Lesions involving the mandibular body and rami

Grade IV

Lesions involving the mandible and maxilla and showing signs of root resorption

Class 1 Lesions involving the mandible and maxillary tuberosity

Class 2 Lesions involving the mandible and anterior maxilla

Class 3 Lesions involving the mandible and entire maxilla

Grade V

The rare, massively growing, aggressive, and extensively deforming juvenile cases involving the maxilla and mandible, and may include the coronoid and condyles

Grade VI

The rare, massively growing, aggressive and extensively deforming juvenile form.

Dental impact

The impact of cherubism lesions on development and eruption of the primary andpermanent dentition varies depending on the time of onset and severity of the expansilelesions. The arrangement of primary teeth can be disturbed. Disruption of the secondary dentition can include absent teeth (mostly molars), rudimentary development of molars, abnormally shaped teeth, partially resorbed roots or delayed and ectopically erupting teeth. Tooth extraction may be needed, especially if teeth are "free-floating" in cherubism lesions or if they become ectopically impacted. In more severe instances, children may require prostheses that need to be adjusted as the child grows or the swelling within the oral cavity changes. Orthodontic treatment is appropriate after growth is completed and when cherubism is regressing. ${ }^{23}$

Cytogenetics

The familial form of cherubism occurs typically in an autosomal dominant trait with mutations in the SH3- domain binding protein 2 (SH3BP2) on chromosome 4p16.3. An autosomal recessive mode of inheritance has been suggested in some instances where signs of cherubism could not be found in carriers of the older generation. Point mutations in the gene coding for SH3BP2 have been identified in 12 of 15 families. All the mutations identified so far are present in exon 9 of the gene and cause amino acid substitutions within a 6-amino acid sequence.

\section{AmelogenesisImperfecta}

Amelogenesisimperfecta (AI) is a group of inherited defects of dental enamel formation that show both clinical and genetic heterogeneity. In its mildest form, AI causes discoloration, while in the most severe presentation the enamel is hypomineralized causing it to be abraded from theteeth shortly after their emergence into the mouth. Both the primary and permanent dentitionsare affected. Enamel findings in AI are highly 
variable, ranging from deficient enamel formation to defects in the mineral and protein content. The four main types of AI were described as follows: hypoplastic, hypomineralized, hypomaturation and with taurodontism. ${ }^{24}$ The AI phenotypes vary widely depending on the specific gene involved, the location and type of mutation, and the corresponding putative change at the protein level. Different inheritance patterns such as X-linked, autosomal dominant and autosomal recessive types have been reported and 14 subtypes of AI were recognized. ${ }^{25}$

\section{Amelogenin}

Amelogenin, the protein product of the AMELX Xq22 and AMELY Yp11 genes, is considered to be critical for normal enamel thickness and structure. At least 14 mutations, 5 nucleotide substitutions; 7 small deletions; and 2 gross deletions have been described in amelogenin gene. This mutation destroys the function of the amelogenin protein completely, producing enamel of normal thickness but poorly mineralized and severe discoloration.

\section{Ameloblastin}

Ameloblastin, also known as amelin, is expressed by the enamel-producing ameloblast cells. The ameloblastin gene is located in chromosome 4, within the critical region for local hypoplastic AI. Ameloblastinbinds specifically to ameloblasts and inhibits cell proliferation of mutant ameloblasts.

\section{Enamelin}

Enamelin, the largest enamel extracellular matrix protein, was initially identified by Fukae, et al(1993). It is produced by ameloblasts, initially during the secretory stage concentrating near theTomes processes. Much lower levels of enamelin expression have been observed in dental pulp, presumably secreted by odontoblasts, and along the forming root. The enamelin gene mutations have been identified in autosomal dominant forms of hypoplastic AI.

\section{Enamelysin (Mmp-20)}

MMP-20, also known as enamelysin, was originally identified by Bartlett et al (1996). Thisenzyme is expressed by ameloblasts and the odontoblasts of the dental papilla. Therefore, MMP-20 is considered a toothspecific metalloproteinase. MMP-20 expression was observed in pathologic tissues such as in calcifying odontogenic cysts, odontogenic tumors and tongue carcinoma. ${ }^{16}$

\section{$>$ DentinogenesisImperfecta}

It was probably first recognized by Barret in 1882. The first published report describing the disorder as an enamel defect was by Talbot as quoted by Witkop. The term 'hereditary opalescent dentin' was first used by Skillen, Finn and Hodges to describe the brown translucent teeth that have an opalescent sheen and are lacking in pulp chambers.Dentinogenesisimperfecta (DGI) is a localized mesodermal dysplasia affecting both the primary and permanent dentitions. Clinically, both dentitions are affected. The color of the teeth varies from brown to blue, and is sometimes described as amber or gray.

Radiographically, the teeth have bulbous crowns and constricted, short roots. Initially, the pulp chambers may be abnormally wide, giving the appearance of 'shell teeth' but they progressively get obliterated. ${ }^{26}$ DGI has been classified by Shields and co-workers into three types:

1. Type I, DGI associated with osteogenesis imperfecta

2. Type II, DGI without osteogenesis imperfect

3. Type III, brandywine type.

\section{Dentinogenesisimperfecta type I}

Individuals with DGI-I also have osteogenesis imperfecta. The teeth of both dentitions are typically amber and translucent and show significant attrition. Radiographically, the teeth have short, constricted roots and dentine hypertrophy leading to pulpal obliteration either before or just after eruption.

\section{Dentinogenesisimperfecta type II}

The dental features of DGI-II are similar to those of DGI-I but penetrance is virtually complete and osteogenesis imperfecta is not a feature. Bulbous crowns are a typical feature with marked cervical constriction. Normal teeth are never found in DGI-II. Sensorineural hearing loss has also been reported as a rare feature of the condition.

\section{Dentinogenesisimperfecta type III}

This is a form of DGI found in a tri-racial population from Maryland and Washington DC known as the Brandywine isolate. The clinical features are variable and resemble those seen in DGI-I and -II but the primary 
teeth show multiple pulp exposures and radiographically, they often manifest "shell" teeth i.e. teeth which appear hollow due to hypotrophy of the dentine. ${ }^{27}$

\section{GENETICS AND PERIODONTAL DISEASES}

Periodontal diseases are a heterogenous group of diseases characterized by varying degrees of pathological changes in periodontium. It results in the destruction of the supporting structures and most of the destructive processes involved are host derived. Periodontal diseases may be broadly grouped into two types, Gingivitis and Periodontitis. ${ }^{28}$

Gingivitis is the inflammation of gingiva in the absence of clinical attachment loss. Periodontitis is an inflammatory disease of the supporting tissues of the teeth caused by specific microorganisms or groups of specific microorganisms, resulting in progressive destruction of periodontal ligament and alveolar bone with pocket formation, recession or both. While microbial and other environmental factors are believed to initiate and modulate periodontal disease progression, there now exist strong supporting data that genetic polymorphisms play a role in the predisposition to and progression of periodontal diseases. ${ }^{29}$

Clinically distinct periodontal infections that can affect young individuals include: 1) dental plaqueinduced gingival diseases; 2) chronic periodontitis; 3) aggressive periodontitis; 4) periodontitis as a manifestation of systemic diseases; and 5) necrotizing periodontal diseases. ${ }^{30}$

\section{$>$ Early Onset Periodontits (Aggressive Periodontitis)}

Aggressive periodontitis was previously classified as early onsetperidontitis. It included pre-pubertal, juvenile and rapidly progressive forms of periodontitis. Aggressive periodontitis can be localized or generalized. Localized aggressive periodontitis have interproximal attachment loss on at least two permanent first molars and incisors, with attachment loss on no more than two teeth other than first molars and incisors. Generalized aggressive periodontitis patients exhibit generalized interproximal attachment loss including at least three teeth that are not first molars and incisors. Along with hypophosphatasia, prepubertal periodontitis appears to be the most commonly encountered cause of premature exfoliation of the primary teeth, especially in girls. Chromosome 11q14 that contains cathepsin C gene has been localized for pre pubertal periodontitis. An IL1B allele has been reported to be in linkage disequilibrium with generalized aggressive periodontitis. ${ }^{31}$

\section{$>$ Gingival Enlargement}

It is the overgrowth of gingiva characterized by an expansion and accumulation of the connective tissue with occasional presence of increased number of cells. Gingival enlargement may result from chronic gingival inflammation. It may occur as a drug-related side effect in some individuals. Calcium channel blockers, phenytoin, cyclosporin have been associated with this adverse effect.

Hereditary gingival enlargement is characterized by a slowly progressive benign enlargement of the gingival tissues. Hereditary gingival fibromatosis is a rare disease of infancy characterized by progressive gingival enlargement of normal color and firm consistency that is non-hemorrhagicand asymptomatic. It results in diastemas, malpositioning of teeth, prominent lips and open lip posture. Three different loci have been associated with hereditary gingival fibromatosis: two mapping to chromosome 2 (GINGF on 2p1-22 and GINGF3 on 2p22.3-p23.3) which do not overlap, and one mapping to chromosome 5 (GINGF2 on 5q13-q22). ${ }^{32}$

\section{GENETIC TESTING}

"Genetic screening" denotes assays undertaken on a population wide basis to identify at-risk individuals. "Genetic testing" denotes assays designed to provide a definitive diagnosis; these are performed because of positive screening results, family history, ethnicity, physical stigmata, or other reasons. Diagnostic genetic testing may be performed on a child with signs or symptoms of a potential genetic condition or for treatment decisions made on the basis of results of pharmacogenetic assays. Genetic testing may also be performed on an asymptomatic child with a positive family history for a specific genetic condition, particularly if early treatment may affect morbidity or mortality.

Available types of testing include:

Newborn screening: Newborn screening is used just after birth to identify genetic disorders that can be treated early in life.

Diagnostic testing: Diagnostic testing is used to diagnose or rule out a specific genetic or chromosomal condition.

Carrier testing: Carrier testing is used to identify people who carry one copy of a gene mutation that, when present in two copies, causes a genetic disorder.

Prenatal diagnosis: Used to detect changes in a fetus's genes or chromosomes before birth.

Predictive and Presymptomatic Testing: Predictive and presymptomatic types of testing are used to detect gene mutations associated with disorders that appear after birth, often later in life. 
Histocompatibility Testing: The human leukocyte antigen (HLA) system comprises the major histocompatibility complex in humans. Genetic testing for HLA matching is most important for bone marrow and less important for solid organs.

Diagnostic tests available: The screening tests currently available include:

Chronic Villus Sampling (CVS) - usually done at 10 to 12 weeks of pregnancy to obtain a sample of the placenta by passing a plastic tube through the vagina and into the uterus or by passing a needle through the abdomen and into the uterus.

Blood Test For Alpha-Fetoprotein (AFP) may be performed at 16 to 18 weeks of pregnancy and is used to indicate the level of AFP, a substance produced by the foetus and passed to the mother's blood.

Amniocentesis may be done at 13 to 18 weeks of pregnancy and is a widely used procedure of obtaining amniotic fluid from the uterus by using a needle to pass through the abdomen. ${ }^{33}$

\section{GENETIC COUNSELLING}

Modern genetic counselling is a communication process between a healthcare professional trained in genetics and an individual or family affected by or at risk for an inherited disorder. The goals of this process include promoting awareness of the medical facts of the condition, understanding the role of heredity in the expression of the condition and its risk of recurrence, discussing the options available for dealing with the disorder, and assisting families in choosing the options that are most appropriate for them. ${ }^{34}$

\section{CONCLUSION}

Genetics is the science of heredity and variation. It plays an important role in determining our individuality. Genetics provides an insight into what makes us humans and what distinguishes each of us as individuals.Often pediatric dentists are the first health care practitioners to document dysmorphic features in a child. It is important for them to have an understanding of molecular genetics because the sensitivity and specificity of molecular-based diagnostics have revolutionized how diseases and disorders are defined. These scientific and technological advances translate into improved health, disease prevention, smarter diagnostics, and innovative therapeutic approaches to craniofacial dysmorphogenesis.

\section{REFRENCES}

[1]. Sturtevant AH. A history of genetics

[2]. Phil McClean. A History of Genetics and Genomics.2011.

[3]. Snustad DP, Simmons MJ. Principles of genetics.5 thedn. Asia;2002.

[4]. Cooper GM, Hausman RE. The cell-A molecular approach.5 th edn.ASM press

[5]. Gardner EJ, Snustad DP. Principles of genetics.7 thedn. Singapore; 1984.

[6]. Vieira AR. Genetics and Caries - Perspectives. Braz Oral Res. 2012; 26(Suppl 1):7-9.

[7]. Goldberg S. The dental arches of identical twins. Dental Cosmos. 1930;72:869-81

[8]. Bordoni N, Dono R, Manfredi C, Allegrotti I. Prevalence of dental caries in twins. J Dent Children 1973;40:440-3

[9]. Mansbridge JN. Heredity and dental caries. J Dent Res. 1959;38:337-47.

[10]. Finn SB, Caldwell RC. Dental caries in twins I. A comparison of caries experience of monozygotic twins, dizygotic twins and unrelated children. Arch Oral Biol. 1963;8:571-85.

[11]. Boraas JC, Messer LB, Till MJ. A genetic contribution to dental caries, occlusion and morphology as demonstrated by twins reared apart. J Dent Res 1988;67:1150-5.

[12]. Klein H, Palmer CE. Studies of dental caries. V. Familial resemblance in caries experience in siblings, Public Health Rep.1938;53:1353-1364.

[13]. Klein H. The family and dental disease. IV. Dental disease experience in parents and offsprings, J Am Dent Assoc. 33:735$743,1946$.

[14]. Li Y, Caufield PW. The fidelity of initial acquisition of mutans streptococci by infants from their mothers .J Dent Res.1995;74(2):681-685.

[15]. Goodman HO, Luke JE, Rosen S, Hackel E. Heritability in dental caries, certain oral microflora and salivary components. Am J Hum Genet. 1959;11:263-73.

[16]. Mcdonald RE, Avery DR. Dentistry for the Child and Adolescent.8 TH edn.2004.

[17]. Fairpo CG. Total caries experience in monozygotic and like-sexed dizygotic twins of Caucasoid origin aged 5 to 15 years. Arch Oral Biol 1979;24:491-4.

[18]. Gorlin RJ, Cohen MM, Levin LS. Syndromes of the Head and Neck 3 rd Edition. NY: Oxford Univ. Press.1990;700-704.

[19]. Korenberg JR et al. Down syndrome phenotypes: The consequences of chromosomal imbalance. ProcNatlAcad Sci.1994;91:49975001.

[20]. Patterson D. The integrated map of human chromosome 21. In Etiology and Pathogenesis of Down Syndrome, Wiley-Liss.1995;4355.

[21]. Kalantar MH: Treatment of cherubism with locally aggressive behavior presenting in adulthood: report of four cases and a proposed new grading system. Journal of Oral and Maxillofacial Surgery. 1998;56(11):1336-42.

[22]. Raposo CE, et al: Two-stage surgical treatment of severe cherubism. Annals of Plastic Surgery. 2007;58(6):645-51.

[23]. Thompson N: Cherubism: familial fibrous dysplasia of the jaws. British Journal of Plastic Surgery. 1959;12:89-103.

[24]. Crawford PJ, Aldred MJ. X-linked amelogenesisimperfecta. Presentation of two kindreds and a review of the literature. Oral Surg Oral Med Oral Pathol. 1992;73(4):449-55

[25]. Li W, Gao C, Yan Y, DenBesten P. X-linked amelogenesisimperfecta may result from decreased formation of tyrosine rich amelogenin peptide (TRAP). Arch Oral Biol. 2003;48(3):177-83. 
[26]. Shields ED, Bixler D, Kafrawy AM: A proposed classification for heritable human dentine defects with a description of a new entity. Arch Oral Biol.1973;18:543-553.

[27]. Pallos D, Hart PS, Cortelli JR, Vian S, Wright JT, Korkko J et al. Novel COL1A1 mutation (G559C) [correction of G599C] associated with mild osteogenesis imperfect and dentinogenesisimperfecta. Arch Oral Biol. 2001;46:459-470

[28]. Bimstein E, Needleman HL, Karimbux N, Dyke TE. Periodontal and gingival health and diseases. United Kingdom: Martin Dunitz Ltd;2001.

[29]. Novak JM. Classification of diseases and conditions affecting the periodontium. In: Newman MG, Takei HH, Klokkevold PR, Carranza FA. Carranza's Clinical Periodontology. 10th edition. Saunders Company. 2006:100-109.

[30]. Kinane DF. Genes and gene polymorphisms associated with periodontal disease. Crit Rev Oral Biol Med. 2003;14(6):430-449.

[31]. Hart TC, Hart PS, Michalec MD et al. Localisation of a gene for prepubertal periodontitis to chromosome 11q14 and identification of a cathepsin C gene mutation. J Med Genet.2000;37:95-101

[32]. Coletta RD, Graner E. Hereditary gingival fibromatosis - a systematic review. J Periodontol 2006;77:753-764

[33]. Ross LF, Saal HM, David KL, Anderson RR. Technical report: ethical and policy issues genetic testing and screening of children. American College of Medical Genetics and Genomics.

[34]. Phillips SE. Genetic counseling. Encyclopedia of life science.2001. 